\title{
Cardiovascular manifestations of Marfan's syndrome: improved evaluation by transoesophageal echocardiography
}

\author{
Iain A Simpson, Mark A de Belder, Tom Treasure, A John Camm, Charles W Pumphrey
}

\begin{abstract}
Objective-To assess the value of transoesophageal echocardiography in patients with Marfan syndrome particularly those with suspected aortic pathology or where conventional transthoracic imaging was suboptimal.

Design and patients-Eleven patients with Marfan syndrome. Seven patients were studied because of suspected aortic dissection and four because of inadequate transthoracic imaging.

Intervention-Transoesophageal echocardiography and colour Doppler flow mapping by a $5 \mathrm{MHz}$ single plane transoesphageal probe.
\end{abstract}

Results-Aortic dissection was identified in six patients with subsequent diagnostic confirmation in all six. No dissection was found in one patient in whom the diagnosis had been suspected clinically. Estimates of aortic root dimensions and assessment of aortic and mitral valve pathology were made in four other patients with inadequate transthoracic imaging.

Conclusions-Transoesophageal echocardiography provides rapid diagnostic information in patients with Marfan syndrome with suspected aortic dissection and enhances the assessment of cardiovascular manifestations of this condition.

(Br Heart f 1993;69:104-108)

The cardiovascular manifestations of Marfan syndrome account for most premature deaths from this condition. ${ }^{1}$ These are usually a result of aortic dissection or rupture of the thoracic aorta or both, or are caused by cardiac failure secondary to progressive aortic regurgitation. The importance of accurately detecting such cardiovascular involvement in patients with Marfan syndrome is highlighted by the $87 \%$ reported cardiovascular mortality and by the excellent results of surgery since the development and widespread use of composite aortic grafts. ${ }^{12}$ Recommendations of the timing of surgery have also emphasised the importance of prophylactic replacement once the ascending aortic has reached a diameter of $6 \mathrm{~cm}$ or greater even in symptom free patients ${ }^{3}$ further stressing the need for accurate and repeatable imaging of the thoracic aorta.

Identification of cardiovascular involvement in Marfan syndrome has been greatly enhanced by the introduction of cross sectional echocardiography. Chest $x$ ray films are unhelpful in this condition as the first part of the ascending aorta adjacent to the aortic annulus cannot be seen. This being the critical region for aortic dilatation in Marfan syndrome, it is not suprising that echocardiography compares favourably with chest $x$ ray film for the detection of aortic root dilation $^{4}$ and echocardiography is also the technique of choice for assessment of mitral valve dysfunction in these patients. ${ }^{5}$ The main cause of death, however, in patients with Marfan syndrome is aortic dissection and rupture, which may be undetected by conventional transthoracic echocardiography. Also, as many of these patients have chest deformities, accurate measurement of the aortic root diameter may not be possible. The introduction of transoesophageal echocardiography ${ }^{6-8}$ has largely overcome these limitations of transthoracic echocardiography yet its role in the investigation of patients with Marfan syndrome has not been established. There is a great attraction for such a repeatable diagnostic imaging technique that can be used on an outpatient basis. Also, the potential to detect rapidly the presence and extent of aortic dissection within the confines of the intensive care unit would confer considerable advantages over other imaging techniques such as computed tomography scanning and magnetic resonance imaging. It was the aim of this study to use transoesphageal echocardiography in patients with Marfan syndrome in whom the diagnosis of aortic dissection was suspected or to provide high resolution diagnostic information in patients where conventional transthoracic imaging was regarded as inadequate.

\section{Patients and methods}

\section{PATIENTS}

Transoesophageal echocardiography was performed in 11 patients, eight men and three women, age range 19-56, mean 35 years. All 11 patients fulfilled the criteria for a diagnosis of Marfan syndrome as laid down by Pyeritz and McKusick. ${ }^{9}$ These criteria included (a) ocular manifestations, (b) characteristic skeletal deformities, (c) cardiovascular manifestations, (d) family history of Marfan syndrome. The table shows individual patient details.

All patients were studied because of a specific indication for transoesophageal echocardiography or because of a suboptimal transthoracic echocardiographic examination. 
Individual patient data

\begin{tabular}{lllll}
\hline Patient No & Age & Sex & Reason for TOE & Abnormalities found by TOE \\
\hline 1 & 32 & M & Poor TTE images & Dilated ascending aorta, MVP \\
2 & 36 & M & Poor TTE images & Dilated ascending aorta, AR, MVP \\
3 & 39 & M & Aortic dissection & No dissection, MVP \\
4 & 19 & M & Aortic dissection & Type II dissection, AR \\
5 & 44 & W & Aortic dissection & Type III aortic dissection, MVP \\
6 & 29 & M & Aortic dissection & Type I dissection, AR, no pericardial effusion \\
7 & 50 & M & Poor TTE images & Dilated ascending aorta, MVP, AR \\
8 & 21 & M & Poor TTE images & Dilated ascending aorta \\
9 & 37 & W & Aortic dissection & Type I dissection, MVP \\
10 & 56 & W & Aortic dissection & Type I dissection, pericardial effusion and \\
11 & 26 & M & Aortic dissection & type I dissection, AR, no pericardial effusion \\
\hline
\end{tabular}

TOE, transoesophageal echardiography; TTE, transthoracic echocardiography; MVP, mitral valve prolapse; AR, aortic regurgitation.

Seven patients were studied for a presumptive clinical diagnosis of aortic dissection and four other patients were studied to assess aortic root dimensions as well as aortic and mitral pathology after suboptimal imaging by conventional transthoracic echocardiography.

\section{METHODS}

Transoesophageal echocardiography was performed with a Hewlett-Packard Sonos 1000 imaging system with a $5 \mathrm{MHz}$ single plane transoesophageal imaging probe. All patients received topical pharyngeal anaesthesia with lignocaine spray and those with suspected aortic dissection were routinely given $2.5-5 \mathrm{mg}$ midazolam intravenously in an attempt to prevent any significant hypertensive response during probe insertion. Midazolam was administered to outpatients only if required for patient tolerance. Transoesophageal echocardiography was performed by a physician experienced in the technique with the patient in the left lateral decubitus position. A trained nurse was always present and full resuscitation facilities were available. Informed consent was obtained in all patients. All patients underwent transthoracic imaging before transoesophageal study.

In patients with suspected aortic dissection, attempts were made to get the most important diagnostic information as soon as possible after probe insertion. Thus the aortic root, ascending aortia, aortic arch, and the entire thoracic descending aortia were imaged first. As a single plane transoesophageal probe was used, the uppermost portion of the ascending aorta and the first third of the aortic arch were obscured by the left main bronchus traversing the imaging plane between the oesophagus and this part of the thoracic aorta. The presence of aortic regurgitation or pericardial effusion was then established followed by detailed inspection of the mitral valve apparatus. Other cardiac structures including both atria, systemic and pulmonary venous connections, and right and left ventricular function were studied at the end of the transoesophageal study in case adverse haemodynamic factors or patient intolerance caused the study to end prematurely. In all other patients a more routine systematic approach to transoesophageal imaging was performed. All images were recorded on half inch video tape for subsequent analysis.

Colour Doppler flow mapping was performed in all cases with a velocity variance or velocity enhanced colour Doppler flow map algorithm with optimum colour Doppler flow mapping system settings that included a large pulse packet size, high line density, narrow colour sector, low pulse repetition frequency, and moderately high gain settings without significant spatial or temporal filtering. System settings were adjusted to allow optimum view of the spatial flow velocities combined with high resolution echocardiographic imaging. Colour Doppler flow mapping was used to identify the true and false lumina as well as the entry and re-entry points in case of aortic dissection, the presence of aortic regur-
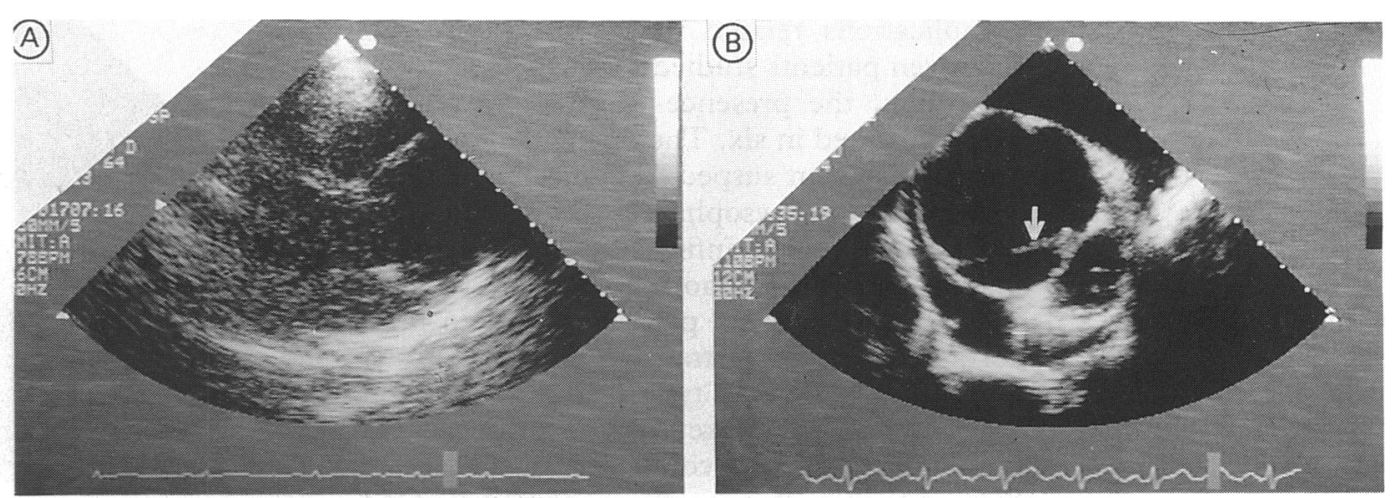

Figure 1 (A) Transthoracic echocardiogram in the parasternal long axis from a patient with suspected aortic dissection. There is no evidence of an intimal tear in the aortic root. (B) Transoesophageal echocardiogram at the level of the aortic root from the same patient. Note the dilated aorta and the presence of an intimal flap (arrowed) within the aortic lumen. The gap in the intimal flap indicates the level of the aortic teart. 

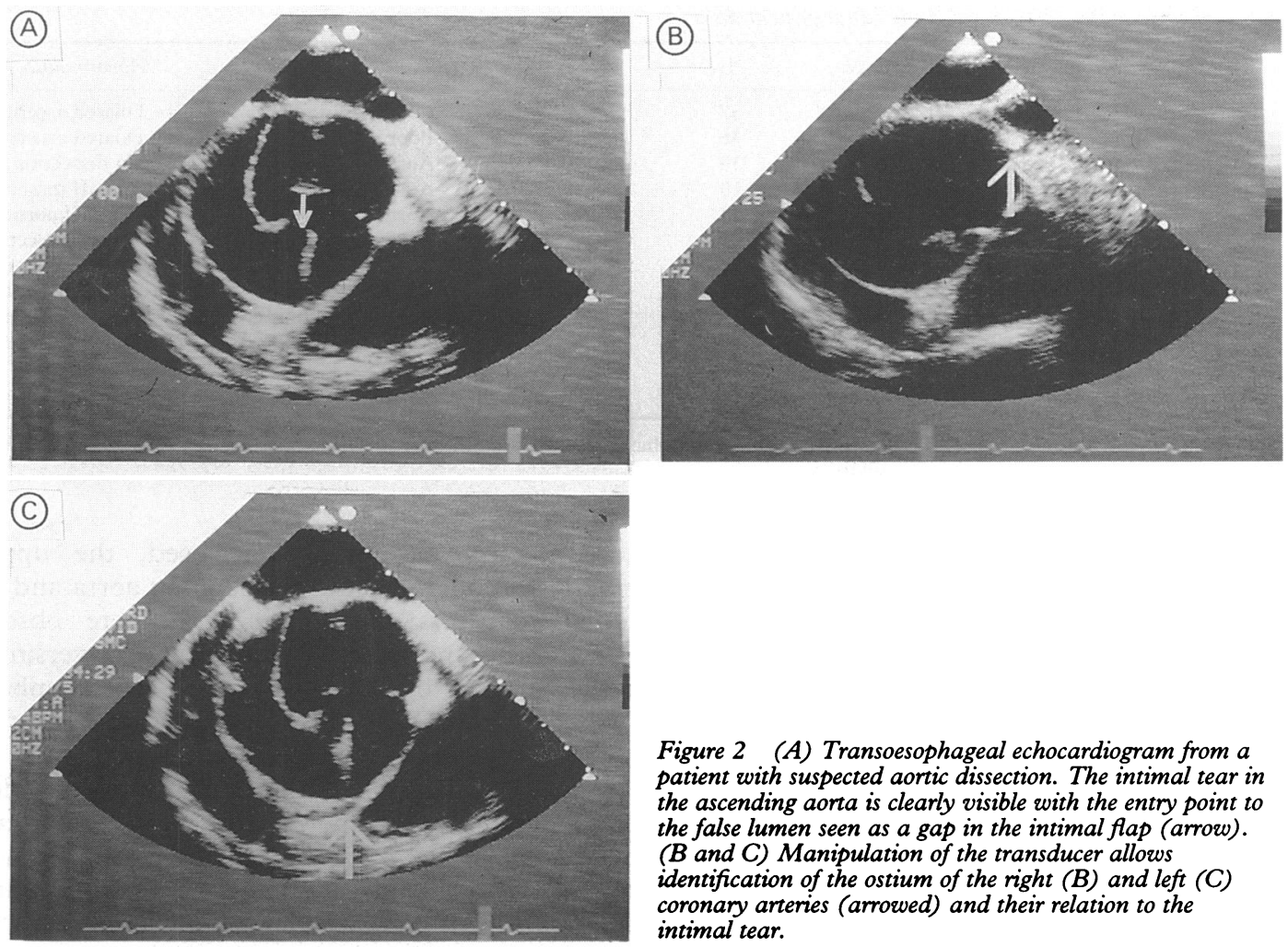

Figure 2 (A) Transoesophageal echocardiogram from a patient with suspected aortic dissection. The intimal tear in the ascending aorta is clearly visible with the entry point to the false lumen seen as a gap in the intimal flap (arrow).

$(B$ and $C$ ) Manipulation of the transducer allows identification of the ostium of the right $(B)$ and left $(C)$ coronary arteries (arrowed) and their relation to the intimal tear.

gitation, and the presence and direction of the jet of mitral regurgitation. The diagnosis of mitral valve prolapse was not based solely on the presence of minor mitral leaflet displacement ${ }^{1011}$ but also on the presence of mitral regurgitation on spectral Doppler or colour Doppler flow mapping, particularly when this was directionally appropriate for the appearance of leaflet prolapse on echocardiographic imaging. In patients in whom the diagnosis of aortic or mitral regurgitation was confirmed on colour Doppler flow mapping, no attempt was made to quantify the severity of the regurgitation based on the size of the regurgitant jet because of the recognised dependence of jet size on both instrumentation and haemodynamic factors. ${ }^{12-14}$

\section{Results}

Transoesophageal echocardiography was well tolerated in all patients and there were no complications related to the procedure. Of the seven patients studied for suspected aortic dissection, the presence of aortic dissection was confirmed in six. The one patient with an aortic dissection suspected clinically who had a normal transoesophageal echocardiogram was subsequently confirmed as having a normal thoracic aorta at aortography.

Of the total of six patients found to have aortic dissection at transoesophageal study, four had a DeBakey type I aortic dissection and one had a DeBakey type II aortic dissection. Diagnosis was confirmed in all five of these patients at surgery or necropsy. One further patient had a DeBakey type III dissection that was subsequently confirmed at computed tomography. In the five patients with a type I or II dissection, transoesophageal echocardiography successfully identified the site of the dissection in all patients. Figure 1(A) shows a transthoracic parasternal long axis view from a patient with suspected aortic dissection but the poor images made diagnostic confirmation impossible. By comparison, fig $1(B)$ is the transoesophageal echocardiogram from the same patient that shows a type I aortic dissection and an intimal flap in the ascending aortia (arrowed) that was not apparent with transthoracic imaging. As well as confirming the diagnosis of aortic dissection, transoesophageal echocardiography was able to determine accurately the entry point of the dissection in the ascending aorta in all patients (fig 2(A)). It was also possible to determine the relation of the dissection to the coronary ostia (fig $2(\mathrm{~B})$ and (C)) by subtle manipulation of the transducer plane. The

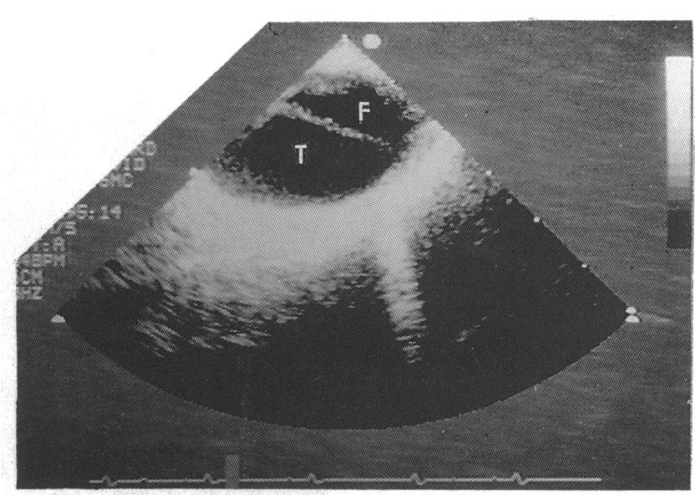

Figure 3 Transoesophageal echocardiogram at the level of the mid-aortic arch. The intimal flap is seen to extend to the level of the transverse aortic arch. $T$, true lumen; $F$, false lumen. 


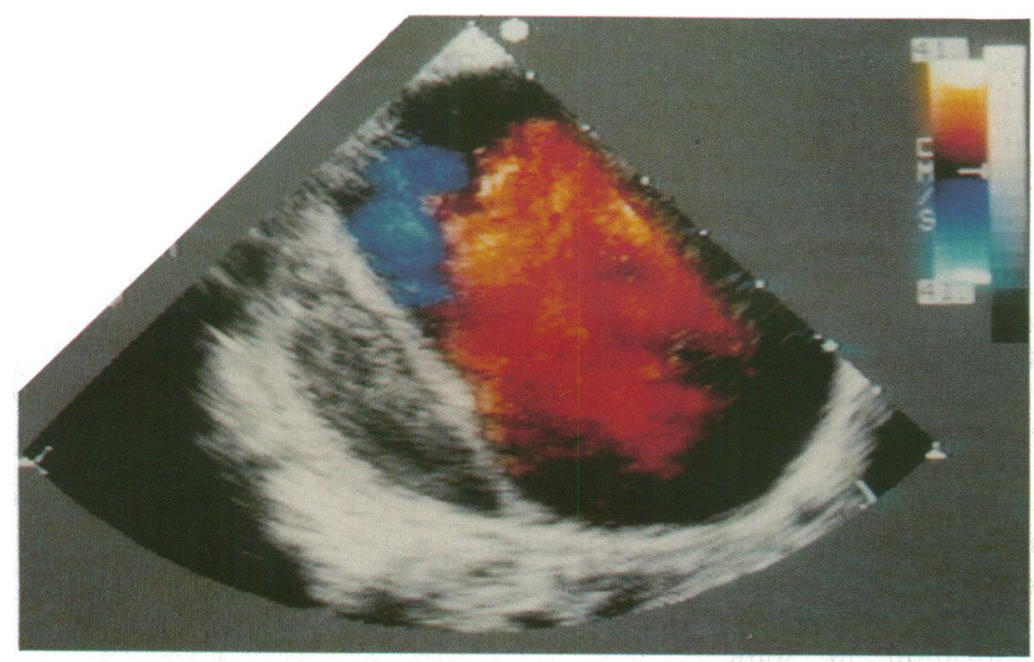

Figure 4 Transoesophageal colour Doppler flow map image at the level of the upper descending aorta from the patient with type III aortic dissection. Thrombus is seen within the smaller false lumen and the presence of good colour flow encoding delineates the larger true lumen. Individual patient data TOE, Transoesophageal echocardiography; TTE,

Transthoracic echocardiography; MVP, mitral valve prolapse; $A R$, aortic regurgitation.

true and false lumina were identified in all cases with colour Doppler flow mapping, which also detected the presence of aortic regurgitation in three patients. The presence of a significant pericardial effusion was seen in two patients. In patients with a type I dissection the intimal flap could be seen extending into the aortic arch (fig 3) and in one patient extension of the dissection into the thoracic descending aortia was clearly seen. Again, colour Doppler flow mapping was used to find the true from the false lumen.

The patient with a type III aortic dissection was found to have an intimal flap with an entry point at the top of the descending aorta just below the origin of the left subclavian artery with the intimal flap extending to below the diaphragm. A thrombus was identified in the false lumen with colour Doppler flow mapping again used to show the true lumen (fig 4).

In the four patients studied because of inadequate transthoracic imaging, transoesophageal images were excellent, enabling accurate measurements of aortic diameter to be made. Aortic root dimensions at the largest diameter segment in the ascending aortia range from 4 to $6 \mathrm{~cm}$ compared with dimensions of 2.5 to $3.5 \mathrm{~cm}$ at the level of the upper descending aorta. Also, of the four patients, three were shown to have anterior leaflet mitral valve prolapse and two had evidence of aortic regurgitation on colour Doppler flow mapping.

\section{Discussion}

The potentially fatal cardiovascular consequences of Marfan syndrome compel early diagnosis of cardiovascular complications to ensure prompt and appropriate patient management. Conventional cross sectional echocardiography has gone a long way to ensure that this is possible, but it is less satisfactory in delineating the extent of aortic dilatation or the presence of an intimal tear in the ascending aorta. In patients who are good imaging subjects it can usually identify the presence of aortic root dilatation, aortic and mitral regurgitation, and the presence of pericardial effusion or tamponade. The aortic arch and descending aorta, however, are rarely seen even in good imaging patients and in those who are particularly difficult to image even the expected information relating to the aortic and mitral valve pathology may be difficult to obtain.

This study shows that transoesophageal echocardiography can provide important diagnostic information relating to cardiovascular involvement in patients with Marfan syndrome and is particularly valuable for those with suspected aortic dissection. Although this diagnosis can be confirmed by other imaging techniques such as computed tomography scanning or magnetic resonance imaging, transoesophageal echocardiography has some advantages. It can be performed within the confines of the intensive care unit and does not therefore require that the patient is moved away from specialised nursing and medical care facilities. Transoesophageal echocardiography can provide diagnostic information in suspected aortic dissection much more rapidly than the other imaging techniques. This is of paramount importance in a condition with such a high early mortality. The difficulties encountered in seeing the upper ascending aorta and the first third of the aortic arch are well recognised in single plane imaging. Although this did not affect the ability of transoesophageal echocardiography to show aortic dissection in this study the potential does exist to miss important pathology in this area. The introduction of biplane and multiplane transoesophageal imaging will obviate this problem to some extent but this technology was not at our disposal at the time of this study.

Transoesophageal echocardiography is increasingly becoming recognised as the technique of choice for investigation of suspected aortic dissection ${ }^{1516}$ but its role in the investigation of patients with Marfan syndrome has not previously been reported. Indeed, it has been suggested that transoesophageal echocardiography can yield diagnostic information that is better than other imaging techniques ${ }^{17}$ including aortography. ${ }^{18,19}$ As transoesophageal echocardiography is increasingly used for the diagnosis of aortic dissection, ${ }^{15-19}$ concerns about its safety are diminishing. Reports of aortic rupture during transoesophageal echocardiography do exist related to the risk of a hypertensive response to probe insertion. ${ }^{20}$ It is therefore essential that all transoesophageal echos be performed by an experienced physician and, if possible, to have blood pressure monitoring and to sedate the patients before study. The need for an experienced operator cannot be overemphasised as the clinical implications are substantial and patients may undergo surgery on the basis of results of transoesophageal echocardiography. 
Many cardiologists and surgeons regard coronary angiography as unnecessary in patients with dissection of the ascending thoracic aorta as the delay in getting such information in a condition with a high early mortality is probably not justified, ${ }^{2122}$ particularly if the diagnosis has aalready been made by transoesophageal echocardiography in the intensive care unit. It should be recognised that coronary angiography may be difficult to obtain in these patients if the false lumen is extensive and it may be unclear whether the coronary ostia originate from the true or the false lumen. Even if coronary angiography is required in these patients, transoesophageal echocardiography can rapidly exclude or confirm the diagnosis and thereby obviate the need for invasive investigation in some patients and select those requiring coronary angiography before emergency surgery. With the exception of the coronary anatomy, transoesophageal echocardiography can provide all the information that the surgeon may need before surgical intervention. As virtually the whole of the thoracic aorta can be seen, the site and extent of the dissection can be recognised as can the relation of the coronary ostia to the dissection flap. The true and false lumina can be identified with colour Doppler flow mapping and the presence of aortic regurgitation or pericardial effusion can be seen.

As a natural extension of transthoracic echocardiography, the transoesophageal approach holds great promise for the follow up of patients who are difficult to image by the transthoracic mode. As progressive aortic dilatation is an important prognostic feature in this condition the ability to measure aortic diameter accurately is crucial and as transoesophageal echocardiography can be routinely performed as an outpatient procedure and is ideally suited to the follow up of Marfan patients. Additionally, echocardiography, by the transthoracic and transoesophageal approaches, remains the non-invasive technique of choice for delineating involvement of the mitral valve and mitral valve apparatus.

Our study shows the role of transoesophageal echocardiography in patients with Marfan syndrome. Although transthoracic echocardiography is often excellent in such patients, the ascending aorta is not always well seen and four of the patients in this study underwent transoesophageal imaging as a result of inadequate transthoracic echocardiography. In such patients, transoesophageal echocardiography should be considered on a regular follow up basis to monitor aortic root dilatation. In this young patient population with Marfan syndrome, transoesophageal echocardiography should now be regarded as the optimal imaging technique for the diagnosis of suspected aortic dissection.

1 Marsalese DL, Moodie DS, Vacante M, Lytle BW, Gill CC, Sterba R, et al. Marfan's syndrome: natural history and long-term follow-up of cardiovascular involvemant. 7 Am Coll Cardiol 1989;14:422- 8.

2 Bentall $H$, DeBono A. A technique for complete replacement of the ascending aorta. Thorax 1968;23:338-9.

Gott VL, Pyeritz RE, MacGovern GJ, Cameron DE, McKusick VA. Surgical treatment of aneurysms of the ascending aorta in the Marfan syndrome: results of ascending aorta in the Marfan syndrome: results of composite-graft

4 Come PC, Fortuin NJ, White RI, McKusick VA Echocardiogrphic assessment of cardiovascular abnormalities in the Marfan syndrome. Am $F$ Med 1983 74:465-74.

5 Pyeritz RE, Wappel MA. Mitral valve dysfunction in the Marfan syndrome: clinical and echocardiographic study of prevalence and natural history. Am $\mathcal{F}$ Med 1983;74 797-807.

6 Seward JB, Khandheria BK, Oh JK, et al. Transoesophageal echocardiography: technique, anatomical correlation, implemention, and clinical applications. Mayo Clin Proc 1988;63:649-80.

7 Abel M, Nishimura R, Callagan M, Rehder K, Ilstrup D Tajik A. Evaluation of intraoperative transoesophageal Tajik A. Evaluation of intraoperative transoesophageal
two-dimensional echocardiography. Anesthesiology 1987 ; two-dim 64 .

8 Mitchell MM, Sutherland GR, Gussenhoven EJ, Taams MA, Roelandt JRTC. Transoesophageal echocardiography. $\mathcal{F}$ Am Soc Echocardiogr 1988;1:362-77.

Pyeritz RE, McKusick VA. The Marfan syndrome: diag nosis and management. $N$ Eng $\mathcal{F}$ Med 1979;300:772-7.

10 Kivokapich J, Child JS, Dadourian BJ, Perloff JK Reassessment of echocardiographic criteria for diagnosis of mitral valve prolapse. Am $\mathcal{C}$ Cardiol 1988;61:131-5.

11 Levine RA, Stathogiannis E, Newell JB, Harrigan P, Weyman AE. Reconsideration of echocardiographic standards for mitral valve prolapse: lack of association between leaflet displacement isolated to the apical four between leaflet displacement isolated to the apical four chamber view and independent echocardiographic 1010-9.

12 Davidoff $R$, Wilkins GT, Thomas JD, Achorn DM Weyman AE. Regurgitant volumes by color flow overestimate injected volumes in an in vitro model (abstract) f Am Coll Cardiol 1987;9:110.

13 Sahn DJ. Instrumentation and physical factors related to visualization of stenotic and regurgitant jets by color Doppler flow mapping. $₹ \mathrm{Am}$ Coll Cardiol 1988;12: 1354-65.

14 Simpson IA, Valdes-Cruz LM, Sahn DJ, Murillo A, Tamura T, Chung KJ. Doppler color flow mapping of simulated in vitro regurgitant jets: Evaluation of the effects of orifice size and hemodynamic variables. $7 \mathrm{Am}$ Coll Cardiol 1989;13:1195-207.

15 Adachi H, Kyo S, Takamoto S, Kimura S, Yokote Y Omoto R. Early diagnosis and surgical intervention of Omoto $R$. Early diagnosis and surgical intervention of acute aortic dissection by transoesophageal color

16 Taams MA, Gussenhoven WJ, Schippers LA, Roelandt J, Van Herwerden LA, Bos E, et al. The value of transoesophageal echocardiography for diagnosis of thoraci aortic pathology. Eur Heart $\mathcal{F}$ 1988;9:1308-16.

17 De Simone $R$, Haberbosch W, Iarussi D, Iacono A Transoesophageal echocardiography for the diagnosis of thoracic aorta aneurysms and dissections. Cardiologia 1990;35:387-90.

18 Erbel R, Mohr-Kahaly S, Rennollet H, Brunier J, Drexler $M$, Wittlich B, et al. Diagnosis of aortic dissection: the value of transoesophageal echocardiography. Thorac value of transoesophageal echoca
Cardiovasc Surg 1987;35:126- 33 .

19 Erbel R, Borner N, Steller D, Brunier J, Thelen M, Peiffer $\mathrm{C}$, et al. Detection of aortic dissection by trans$\mathrm{C}, e t$ al. Detection of aortic dissection by trans-
oesophageal echocardiography. Br Heart $f$ 1987;58. 45-51.

20 Silvey SV, Stoughton TL, Pearl W, Collazo WA, Belbe RJ. Rupture of the outer partition of aortic dissection during transoesophageal echocardiography. $A m \quad \mathcal{F}$ Cardiol 1991;68:286-7.

21 Kern MJ, Serota H, Callicoat $\mathrm{P}$, et al. Use of coronary arteriography in the preoperative management. of patients undergoing urgent repair of the thoracic aorta. Am Heart $\mathcal{F} 1990$; 119:143-8.

22 Treasure T, Raphael MJ. Investigation of suspected aortic dissection of the thoracic aorta. Lancet 1991;338:490-5. 